\title{
The ternary Goldbach problem in arithmetic progressions
}

\author{
by \\ JiANYA LiU and TAO ZHAN (Jinan)
}

For a large odd integer $N$ and a positive integer $r$, define $\mathbf{b}=\left(b_{1}, b_{2}, b_{3}\right)$ and

$\mathcal{B}(N, r)=\left\{\mathbf{b} \in \mathbb{N}^{3}: 1 \leq b_{j} \leq r,\left(b_{j}, r\right)=1\right.$ and $\left.b_{1}+b_{2}+b_{3} \equiv N(\bmod r)\right\}$.

It is known that

$$
\# \mathcal{B}(N, r)=r^{2} \prod_{\substack{p|r \\ p| N}} \frac{(p-1)(p-2)}{p^{2}} \prod_{\substack{p \mid r \\ p \nmid N}} \frac{p^{2}-3 p+3}{p^{2}} .
$$

Let $\varepsilon>0$ be arbitrary and $R=N^{1 / 8-\varepsilon}$. We prove that for all positive integers $r \leq R$, with at most $O\left(R \log ^{-A} N\right)$ exceptions, the Diophantine equation

$$
\left\{\begin{array}{l}
N=p_{1}+p_{2}+p_{3}, \\
p_{j} \equiv b_{j}(\bmod r), \quad j=1,2,3,
\end{array}\right.
$$

with prime variables is solvable whenever $\mathbf{b} \in \mathcal{B}(N, r)$, where $A>0$ is arbitrary.

1. Introduction and statement of results. For given odd integers $N$ we shall be concerned with the solubility of the equation

$$
N=p_{1}+p_{2}+p_{3}
$$

in prime variables $p_{j}$; this is known as the ternary Goldbach problem. Hardy and Littlewood [HL] proved in 1923 that subject to the generalized Riemann hypothesis (GRH hereafter) the number $J(N)$ of solutions of (1.1) satisfies

1991 Mathematics Subject Classification: 11P32, 11L07.

Key words and phrases: ternary Goldbach problem, exponential sum over primes in arithmetic progressions, mean-value theorem.

Project supported by the Trans-Century Training Programme Foundation for the Talents by the State Education Commission and the National Natural Science Foundation of China. 
an asymptotic formula

$$
J(N)=\sigma(N) \frac{N^{2}}{2 \log ^{3} N}(1+o(1)) .
$$

Here $\sigma(N)$ is the singular series, and one has $\sigma(N) \gg 1$ for odd $N$. In 1937 Vinogradov [Vi] obtained for the first time a nontrivial estimate of exponential sums over primes, and managed to establish (1.2) unconditionally.

Since 1923, many authors have considered the corresponding problems with restrictive conditions posed on the three prime variables in (1.1). One of these generalizations was given by Rademacher $[R]$ in 1926 . For a positive integer $r$, define $\mathbf{b}=\left(b_{1}, b_{2}, b_{3}\right)$ and

$$
\begin{aligned}
& \mathcal{B}(N, r) \\
& =\left\{\mathbf{b} \in \mathbb{N}^{3}: 1 \leq b_{j} \leq r,\left(b_{j}, r\right)=1 \text { and } b_{1}+b_{2}+b_{3} \equiv N(\bmod r)\right\} .
\end{aligned}
$$

Then, according to Liu and Tsang [LT],

$$
\# \mathcal{B}(N, r)=r^{2} \prod_{\substack{p|r \\ p| N}} \frac{(p-1)(p-2)}{p^{2}} \prod_{\substack{p \mid r \\ p \nmid N}} \frac{p^{2}-3 p+3}{p^{2}} .
$$

Rademacher $[\mathrm{R}]$ showed, subject to GRH, that if $r$ is a fixed positive integer, and $J(N ; r, \mathbf{b})$ the number of solutions of the equation

$$
\left\{\begin{array}{l}
N=p_{1}+p_{2}+p_{3}, \\
p_{j} \equiv b_{j}(\bmod r), \quad j=1,2,3,
\end{array}\right.
$$

then we have, for odd $N$ and all $\mathbf{b} \in \mathcal{B}(N, r)$,

$$
J(N ; r, \mathbf{b})=\sigma(N ; r) \frac{N^{2}}{2 \log ^{3} N}(1+o(1)),
$$

and the singular series $\sigma(N ; r)$ satisfies

$$
\begin{aligned}
\sigma(N ; r)= & \frac{C(r)}{r^{2}} \prod_{p \mid r} \frac{p^{3}}{(p-1)^{3}+1} \prod_{\substack{p \mid N \\
p \nmid r}} \frac{(p-1)\left((p-1)^{2}-1\right)}{(p-1)^{3}+1} \\
& \times \prod_{p>2}\left(1+\frac{1}{(p-1)^{3}}\right) \gg 1,
\end{aligned}
$$

where $p>2$ throughout, $C(r)=2$ for odd $r$, and $C(r)=8$ for even $r$. Following the work of Vinogradov [Vi], several authors established Rademacher's result unconditionally; see for example Ayoub [A] and Zulauf [Zu].

The arguments of $[\mathrm{A}]$ and $[\mathrm{Zu}]$ with some minor modifications actually give (1.6) for all $r \leq \log ^{A} N$, where $A>0$ is arbitrary. A natural problem is whether (1.6) is still true for larger $r$. The purpose of the present paper is to give a result in this direction. We prove that (1.6) is true for almost 
all positive moduli $r \leq N^{1 / 8-\varepsilon}$ and all $\mathbf{b} \in \mathcal{B}(N, r)$. Precisely speaking, we have the following

TheOREm 1. Let $N$ be a fixed large odd integer, $\varepsilon>0$ arbitrarily small and

$$
R=N^{1 / 8-\varepsilon} .
$$

Let also $A>0$ be arbitrary. For a positive integer $r$, define $\mathcal{B}(N, r)$ as in (1.3). Then for all positive integers $r \leq R$, with at most $O\left(R \log ^{-A} N\right)$ exceptions, the Diophantine equation (1.5) with prime variables is solvable whenever $\mathbf{b} \in \mathcal{B}(N, r)$, and the number of solutions is given by (1.7).

The above result is a consequence of the following mean-value theorem.

Theorem 2. Let $N$ be a fixed large odd integer, $\varepsilon>0$ arbitrarily small and

$$
R=N^{1 / 8-\varepsilon} .
$$

Let also $A>0$ be arbitrary. For a positive integer $r$, define $\mathcal{B}(N, r)$ as in (1.3). Then

$$
\begin{aligned}
\sum_{r \leq R} r \max _{\mathbf{b} \in \mathcal{B}(N, r)} \mid & \sum_{\substack{N=n_{1}+n_{2}+n_{3} \\
n_{j} \equiv b_{j}(\bmod r)}} \Lambda\left(n_{1}\right) \Lambda\left(n_{2}\right) \Lambda\left(n_{3}\right)-\sigma(N ; r) \frac{N^{2}}{2} \mid \\
& \ll N^{2} \log ^{-A} N,
\end{aligned}
$$

where $\Lambda(n)$ denotes the von Mangoldt function.

R e mark. If the $r$ 's in the theorems are restricted to primes, then the exponent $1 / 8$ can be improved to $3 / 20$. This improvement is useful in studying the ternary Goldbach problem with the three prime summands restricted to a thin subset of primes. This problem has been investigated in another paper [Li].

Since the derivation of Theorem 1 from Theorem 2 is immediate, we give it here.

Proof of Theorem 1 . Let $E(R)$ be the set of positive integers $r \leq R$ for which

$$
\max _{\mathbf{b} \in \mathcal{B}(N, r)}\left|\sum_{\substack{N=n_{1}+n_{2}+n_{3} \\ n_{j} \equiv b_{j}(\bmod r)}} \Lambda\left(n_{1}\right) \Lambda\left(n_{2}\right) \Lambda\left(n_{3}\right)-r \sigma(N ; r) \frac{N^{2}}{2}\right|>\frac{r}{\varphi^{3}(r)} \cdot \frac{N^{2}}{\log N} .
$$

Then one deduces from Theorem 2 that

$$
\sum_{r \in E(R)} \frac{r^{2}}{\varphi^{3}(r)} \leq \log ^{-A} N
$$

for arbitrary $A>0$, and consequently, 


$$
\# E(R)=\sum_{r \in E(R)} 1 \leq R \sum_{r \in E(R)} \frac{r^{2}}{\varphi^{3}(r)} \ll R \log ^{-A} N .
$$

Since

$$
\frac{r}{\varphi^{3}(r)} \ll \sigma(N ; r) \ll \frac{r}{\varphi^{3}(r)},
$$

one sees that (1.6) is true for all $r \notin E(R)$ and all $\mathbf{b} \in \mathcal{B}(N, r)$. This completes the proof of Theorem 1.

Now it remains to establish Theorem 2.

The proof of Theorem 2 is motivated by a paper of Wolke [W], which contains several new ideas to study the problem under consideration and the ternary Goldbach problem with the prime summands restricted to a thin subset of prime numbers. His method actually gave Theorem 2 for almost all prime moduli $r=p \leq N^{1 / 11}$.

The basic tool of our proof, as can be expected, is the circle method. On the minor arcs, one needs a nontrivial estimate for exponential sums over primes in arithmetic progressions to every individual and large modulus $r$. All known results of this kind are, however, nontrivial when the choice of minor arcs is very "thin". Consequently, the major arc is much "larger" than usual. By defining the major and minor arcs in this way, the minor arcs can then be treated easily by a result of Balog and Perelli [BP] on exponential sums over primes in an arithmetic progression (see Lemma 1 below). The main difficulty of the proof comes from the major arcs, where we use the following ideas:

(a) The starting point is Lemma 2 in $\S 2$, where we establish a new formula for

$$
\sum_{\substack{n \leq N \\ n \equiv b(\bmod r)}} \Lambda(n) e(n \alpha)
$$

in terms of Dirichlet characters. It plays a similar role as the formula

$$
\sum_{n \leq N} \Lambda(n) e\left(n\left(\frac{a}{q}+\lambda\right)\right)=\frac{1}{\varphi(q)} \sum_{\chi \bmod q} G(a, \bar{\chi}) \sum_{n \leq N} \Lambda(n) e(n \lambda)
$$

does in the treatment of the original ternary Goldbach problem, where $G(a, \chi)$ is the Gaussian sum defined as

$$
G(a, \chi)=\sum_{n=1}^{k} \chi(n) e\left(\frac{a n}{k}\right)
$$

Consequently, a generalization of the Gaussian sum, namely $G\left(b, f, m, \chi_{g}, k\right)$ defined as in $\S 2$, occurs. We need upper estimates for $G\left(b, f, m, \chi_{g}, k\right)$, and these are established in $\S 3$. 
(b) The treatment of the major arcs eventually reduces to the following form of mean-value estimates for exponential sums over primes:

Theorem 3. For any $A>0$, there exists a constant $E=E(A)>0$ such that if

$$
1 \leq K \leq x^{1 / 3} L^{-E}, \quad \theta=K^{-3} L^{-E}
$$

then

$$
\sum_{q \leq K} \max _{y \leq x} \max _{(a, q)=1} \max _{|\lambda| \leq \theta}\left|\sum_{n \leq y} \Lambda(n) e\left(n\left(\frac{a}{q}+\lambda\right)\right)-\frac{\mu(q)}{\varphi(q)} \sum_{n \leq y} e(n \lambda)\right| \ll x L^{-A} .
$$

In $\S 4$, a general result (Theorem 4) containing this theorem is established. These mean-value estimates play important roles in the proof of Theorem 2 , and the exponent $1 / 8$ results from them.

It should be mentioned that Maier and Pomerance $[\mathrm{MP}]$, Balog $[\mathrm{B}]$ and Mikawa [Mi] studied the distribution of prime twins with one of them in arithmetic progressions. Their methods can deal with the binary Goldbach problem with one of the summands in arithmetic progressions, but we cannot apply them to the problem considered in the present paper.

We use standard notations in number theory. In particular, the letter $r$ in the sequel stands always for positive integers, while $L$ for $\log N$ except in $\S 4$ where $L=\log x$. The letter $\delta$ denotes a sufficiently small positive number, whose value may vary in different occurrences. For example, we can write

$$
N^{\delta} L^{5} \ll N^{\delta}, \quad N^{\delta} N^{\delta} \ll N^{\delta} .
$$

The expression $r \sim R$ means $\frac{1}{2} R<r \leq R$. A Dirichlet character $\chi \bmod q$ will be written as $\chi_{q}$ if necessary.

\section{Outline of the proof of Theorem 2. Let}

$$
R \leq N^{1 / 8-\varepsilon},
$$

and

$$
P=R^{2} L^{3 C}, \quad Q=N R^{-2} L^{-4 C} ;
$$

the constant $C$ will be specified later. For each positive integer $r \sim R$, the major arc of the circle method is defined as

$$
E_{1}(R)=\bigcup_{q \leq P} \bigcup_{\substack{a=1 \\(a, q)=1}}^{q}\left[\frac{a}{q}-\frac{1}{q Q}, \frac{a}{q}+\frac{1}{q Q}\right]
$$

Since $2 P<Q$, no two major arcs intersect. The minor arc is defined as

$$
E_{2}(R)=\left[\frac{1}{Q}, 1+\frac{1}{Q}\right]-E_{1}(R)
$$


Write $\alpha \in[0,1]$ in the form

$$
\alpha=a / q+\lambda, \quad 1 \leq a \leq q, \quad(a, q)=1 .
$$

It follows from Dirichlet's lemma on rational approximations that

$$
E_{2}(R)=\{\alpha: P<q \leq Q,|\lambda| \leq 1 /(q Q)\} .
$$

Let $\Lambda(n)$ be the von Mangoldt function, $e(\alpha)=e^{2 \pi i \alpha}$ as usual, and

$$
S(\alpha ; r, b)=\sum_{\substack{n \leq N \\ n \equiv b(\bmod r)}} \Lambda(n) e(n \alpha) .
$$

Then the statement of Theorem 2 is equivalent to that, for arbitrary $A>0$,

$$
\begin{array}{r}
\sum_{r \sim R} r \max _{\mathbf{b} \in \mathcal{B}(N, r)}\left|\int_{0}^{1} S\left(\alpha ; r, b_{1}\right) S\left(\alpha ; r, b_{2}\right) S\left(\alpha ; r, b_{3}\right) e(-N \alpha) d \alpha-\sigma(N ; r) \frac{N^{2}}{2}\right| \\
\ll N^{2} L^{-A} .
\end{array}
$$

It thus suffices to prove

$$
\begin{aligned}
\sum_{r \sim R} r \max _{\mathbf{b} \in \mathcal{B}(N, r)} \mid \int_{E_{1}(R)} S\left(\alpha ; r, b_{1}\right) S\left(\alpha ; r, b_{2}\right) & S\left(\alpha ; r, b_{3}\right) e(-N \alpha) d \alpha \\
& -\sigma(N ; r) \frac{N^{2}}{2} \mid \ll N^{2} L^{-A},
\end{aligned}
$$

and

$$
\begin{aligned}
& \sum_{r \sim R} r \max _{\mathbf{b} \in \mathcal{B}(N, r)}\left|\int_{E_{2}(R)} S\left(\alpha ; r, b_{1}\right) S\left(\alpha ; r, b_{2}\right) S\left(\alpha ; r, b_{3}\right) e(-N \alpha) d \alpha\right| \\
& \ll N^{2} L^{-A} .
\end{aligned}
$$

The estimate of $S(\alpha ; r, b)$ with $(b, r)=1$ on the minor arcs is given in the following lemma.

Lemma 1. Let $A>0$ be arbitrary and $\alpha \in E_{2}(R)$. If $C$ is sufficiently large, then

$$
S(\alpha ; r, b) \ll \frac{N}{r \log ^{A} N}
$$

uniformly for $r \sim R$.

Proof. We need the following result of Balog and Perelli [BP]: For $M \leq N$ and $h=(r, q)$,

$$
\sum_{\substack{n \leq M \\ n \equiv b(\bmod r)}} \Lambda(n) e\left(\frac{a}{q} n\right) \ll L^{3}\left(\frac{h N}{r q^{1 / 2}}+\frac{q^{1 / 2} N^{1 / 2}}{h^{1 / 2}}+\frac{N^{4 / 5}}{r^{2 / 5}}\right) .
$$


(A similar result was also obtained by Lavrik [La].) Now the desired estimate can be easily derived from (2.8) via partial summation.

We can now give

Pro of of (2.6). It follows from Lemma 1 that the integral over $E_{2}(R)$ is

$$
\begin{aligned}
& \int_{E_{2}(R)} S\left(\alpha ; r, b_{1}\right) S\left(\alpha ; r, b_{2}\right) S\left(\alpha ; r, b_{3}\right) e(-N \alpha) d \alpha \\
& \quad \ll \max _{\alpha \in E_{2}(R)}\left|S\left(\alpha ; r, b_{1}\right)\right|\left(\int_{0}^{1}\left|S\left(\alpha ; r, b_{2}\right)\right|^{2} d \alpha\right)^{1 / 2}\left(\int_{0}^{1}\left|S\left(\alpha ; r, b_{3}\right)\right|^{2} d \alpha\right)^{1 / 2} \\
& \\
& \quad \ll \frac{N^{2}}{r^{2} L^{A+1}},
\end{aligned}
$$

uniformly for $r \sim R$. Hence the quantity on the left-hand side of (2.6) is $\ll N^{2} L^{-A}$, which proves $(2.6)$.

Theorem 2 now reduces to (2.5), which will be established in the following four sections.

The starting point of the proof of (2.5) is Lemma 2 below, which transforms the exponential sum $S(\alpha ; r, b)$ into character sums. To state the lemma, we need some more notations.

Let $d, f, g, k, m$ be fixed positive integers, and $\chi_{g}$ a Dirichlet character $\bmod g$. Define

$$
G\left(d, f, m, \chi_{g}, k\right)=\sum_{\substack{n=1 \\(n, k)=1 \\ n \equiv f(\bmod d)}}^{k} \chi(n) e(m n / k) .
$$

Obviously, this is a generalization of the Gaussian sum $G(m, \chi)$.

For positive integers $r$ and $q$, let

$$
h=(r, q) .
$$

Then $r, q$ and $h$ can be written as

$$
\begin{aligned}
& r=p_{1}^{\alpha_{1}} \ldots p_{s}^{\alpha_{s}} r_{0}, \quad\left(p_{j}, r_{0}\right)=1, \\
& q=p_{1}^{\beta_{1}} \ldots p_{s}^{\beta_{s}} q_{0}, \quad\left(p_{j}, q_{0}\right)=1, \\
& h=p_{1}^{\gamma_{1}} \ldots p_{s}^{\gamma_{s}}
\end{aligned}
$$

where $\alpha_{j}, \beta_{j}$ and $\gamma_{j}$ are positive integers with $\gamma_{j}=\min \left(\alpha_{j}, \beta_{j}\right), j=1, \ldots, s$. Define

$$
h_{1}=p_{1}^{\delta_{1}} \ldots p_{s}^{\delta_{s}},
$$

where $\delta_{j}=\alpha_{j}$ or 0 according as $\alpha_{j}=\gamma_{j}$ or not. Then $h_{1} \mid h$. Write

$$
h_{2}=h / h_{1} \text {. }
$$


Then

$$
h_{1} h_{2}=h, \quad\left(h_{1}, h_{2}\right)=1, \quad\left(\frac{r}{h_{1}}, \frac{q}{h_{2}}\right)=1 .
$$

Lemma 2. Let $a, q, r$ be positive integers, and $h, h_{1}, h_{2}$ defined as in (2.10), (2.11) and (2.12) respectively so that (2.13) holds. Then

$$
\begin{aligned}
S\left(\frac{a}{q}+\lambda ; r, b\right)= & \frac{1}{\varphi\left(r / h_{1}\right) \varphi\left(q / h_{2}\right)} \sum_{\xi \bmod r / h_{1}} \bar{\xi}(b) \sum_{\eta \bmod q / h_{2}} G(h, b, a, \bar{\eta}, q) \\
& \times \sum_{n \leq N} \xi \eta(n) \Lambda(n) e(n \lambda)+O\left(L^{2}\right),
\end{aligned}
$$

where $G(h, b, a, \bar{\eta}, q)$ is defined as in $(2.9)$.

Proof. It is easily seen that

$$
S\left(\frac{a}{q}+\lambda ; r, b\right)=\sum_{\substack{c=1 \\(c, q)=1}}^{q} e\left(\frac{a c}{q}\right) \sum_{\substack{n \leq N \\ n \equiv b(\bmod r) \\ n \equiv c(\bmod q)}} \Lambda(n) e(n \lambda) .
$$

The inner sum is empty unless $c \equiv b(\bmod h)$; we can therefore add the restriction $c \equiv b(\bmod h)$ to the sum over $c$. On the other hand, under the condition $c \equiv b(\bmod h)$, the simultaneous congruences

$$
n \equiv b(\bmod r), \quad n \equiv c(\bmod q)
$$

are equivalent to

$$
n \equiv b\left(\bmod r / h_{1}\right), \quad n \equiv c\left(\bmod q / h_{2}\right)
$$

according to (2.13). And consequently,

$$
\begin{aligned}
S\left(\frac{a}{q}+\lambda ; r, b\right)= & \sum_{\substack{c=1 \\
(c, q)=1 \\
c \equiv b(\bmod h)}}^{q} e\left(\frac{a c}{q}\right) \sum_{\substack{n \leq N \\
n \equiv b(\bmod r) \\
n \equiv c(\bmod q)}} \Lambda(n) e(n \lambda) \\
= & \sum_{\substack{c=1 \\
(c, q)=1 \\
c \equiv b(\bmod h)}}^{q} e\left(\frac{a c}{q}\right) \sum_{\substack{n \leq N \\
n \equiv b\left(\bmod r / h_{1}\right) \\
n \equiv c\left(\bmod q / h_{2}\right)}} \Lambda(n) e(n \lambda) .
\end{aligned}
$$

Introducing the Dirichlet characters $\xi \bmod r / h_{1}$ and $\eta \bmod q / h_{2}$, one has

$$
S\left(\frac{a}{q}+\lambda ; r, b\right)=\frac{1}{\varphi\left(r / h_{1}\right) \varphi\left(q / h_{2}\right)} \sum_{\substack{c=1 \\(c, q)=1 \\ c \equiv b(\bmod h)}}^{q} e\left(\frac{a c}{q}\right) \sum_{\xi \bmod r / h_{1}} \bar{\xi}(b)
$$




$$
\begin{aligned}
& \times \sum_{\eta \bmod q / h_{2}} \bar{\eta}(c) \sum_{n \leq N} \xi \eta(n) \Lambda(n) e(n \lambda)+O\left(L^{2}\right) \\
= & \frac{1}{\varphi\left(r / h_{1}\right) \varphi\left(q / h_{2}\right)} \sum_{\xi \bmod r / h_{1}} \bar{\xi}(b) \sum_{\eta \bmod q / h_{2}} G(h, b, a, \bar{\eta}, q) \\
& \times \sum_{n \leq N} \xi \eta(n) \Lambda(n) e(n \lambda)+O\left(L^{2}\right) .
\end{aligned}
$$

This proves the lemma.

3. The generalized Gaussian sum $G\left(d, f, m, \chi_{g}, k\right)$. Let $d, f, g, m, k$ be fixed positive integers, and $\chi \bmod g$ a Dirichlet character. The purpose of this section is to give upper estimates for the sum $G\left(d, f, m, \chi_{g}, k\right)$ defined as in (2.9).

The main result of this section is the following

LemmA 3. Let $d|k, g| k$ and $(m, k)=(f, k)=1$. Let also $\chi \bmod g$ be induced by the primitive character $\chi^{*} \bmod g^{*}$. Then

$$
\left|G\left(d, f, m, \chi_{g}, k\right)\right| \leq g^{* 1 / 2} .
$$

In the special case $g=k$, define

$$
G(d, f, m, \chi)=G\left(d, f, m, \chi_{k}, k\right)=\sum_{\substack{n=1 \\ n \equiv f(\bmod d)}}^{k} \chi(n) e(m n / k) .
$$

Then Lemma 3 is a consequence of the following

Lemma 4. Let $d \mid k$ and $(m, k)=(f, k)=1$. Let also $\chi \bmod k$ be induced by the primitive character $\chi^{*} \bmod k^{*}$. Then

$$
|G(d, f, m, \chi)| \leq k^{* 1 / 2} .
$$

Now we derive Lemma 3 from Lemma 4.

Proof of Lemma 3 . Let $\chi_{k}^{0}$ be the principal character $\bmod k$. Then $\chi_{g} \chi_{k}^{0}$ is a character $\bmod k$, and consequently,

$$
G\left(d, f, m, \chi_{g}, k\right)=G\left(d, f, m, \chi_{g} \chi_{k}^{0}\right) .
$$

The desired result follows from Lemma 4 on noting that $\chi_{g} \chi_{k}^{0} \bmod k$ is also induced by the primitive character $\chi^{*} \bmod g^{*}$.

It remains to prove Lemma 4 . To this end, we investigate $G(d, f, m, \chi)$ for some special characters $\chi$ mod $k$ in the following Lemmas $5-7$. The proof of Lemma 4 will then be given at the end of this section. 
Lemma 5. Let $d \mid k$, and $\chi \bmod k$ be primitive. Then

$$
G(d, f, m, \chi)=\frac{k}{d} \cdot \frac{1}{\tau(\bar{\chi})} e\left(\frac{m f}{k}\right) G\left(\frac{k}{d},-m, f, \chi\right),
$$

and consequently,

$$
|G(d, f, m, \chi)| \leq k^{1 / 2}
$$

Here and in the sequel $\tau(\chi)$ is defined by

$$
\tau(\chi)=\sum_{n=1}^{k} \chi(n) e(n / k) .
$$

Proof. Making the substitution $n=j d+f$, one sees that

$$
\begin{aligned}
G(d, f, m, \chi) & =\sum_{j=1}^{k / d} \chi(j d+f) e\left(\frac{m(j d+f)}{k}\right) \\
& =e\left(\frac{m f}{k}\right) \sum_{j=1}^{k / d} \chi(j d+f) e\left(\frac{m j}{k / d}\right) .
\end{aligned}
$$

Now we appeal to the identity

$$
\chi(a)=\frac{1}{\tau(\bar{\chi})} \sum_{n=1}^{k} \bar{\chi}(n) e\left(\frac{a n}{k}\right),
$$

which holds for the primitive character $\chi \bmod k$. Therefore,

$$
\begin{aligned}
\sum_{j=1}^{k / d} \chi(j d+f) e\left(\frac{m j}{k / d}\right) & =\frac{1}{\tau(\bar{\chi})} \sum_{n=1}^{k} \bar{\chi}(n) e\left(\frac{f n}{k}\right) \sum_{j=1}^{k / d} e\left(\frac{n j d}{k}\right) e\left(\frac{m j}{k / d}\right) \\
& =\frac{1}{\tau(\bar{\chi})} \sum_{n=1}^{k} \bar{\chi}(n) e\left(\frac{f n}{k}\right) \sum_{j=1}^{k / d} e\left(\frac{(n+m) j}{k / d}\right) .
\end{aligned}
$$

The inner sum equals $k / d$ or 0 according as $n+m \equiv 0(\bmod k / d)$ or not. Hence the right-hand side above is equal to

$$
\frac{k}{d} \cdot \frac{1}{\tau(\bar{\chi})} \sum_{\substack{n=1 \\ n \equiv-m(\bmod k / d)}}^{k} \bar{\chi}(n) e\left(\frac{f n}{k}\right)=\frac{k}{d} \cdot \frac{1}{\tau(\bar{\chi})} G\left(\frac{k}{d},-m, f, \bar{\chi}\right) .
$$

This in combination with (3.4) gives (3.2).

The inequality (3.3) follows from the well-known fact that $|\tau(\chi)|=k^{1 / 2}$ and the trivial estimate $|G(k / d,-m, f, \bar{\chi})| \leq d$. This completes the proof of the lemma. 
Lemma 6. Let $d \mid k,(m, k)=1$ and $\chi \bmod k$ be induced by the primitive character $\chi^{*} \bmod k^{*}$. If $k^{*}$ satisfies

$$
p|k \Rightarrow p| k^{*}
$$

then

$$
|G(d, f, m, \chi)| \leq k^{* 1 / 2} .
$$

Proof. From the assumption of the lemma, one deduce that

$$
G(d, f, m, \chi)=\sum_{\substack{n=1 \\ n \equiv f(\bmod d)}}^{k} \chi^{*}(n) e(m n / k) .
$$

The following argument is divided into 2 cases.

Special case. We start from the simplest case where $k=p^{\alpha}$ for some prime $p$ and positive integer $\alpha$. Since $k^{*} \mid k$ and $d \mid k$, we can suppose that $k^{*}=p^{\beta}$ and $d=p^{\gamma}$, where $\beta$ and $\gamma$ are integers satisfying $1 \leq \beta \leq \alpha$ and $0 \leq \gamma \leq \alpha$. It is obvious that one has either $k^{*} \mid d$ or $d \mid k^{*}$.

If $k^{*} \mid d$, then on setting $n=d u+f$ the above sum becomes

$$
\begin{aligned}
G(d, f, m, \chi) & =\sum_{u=1}^{k / d} \chi^{*}(u d+f) e\left(\frac{m(u d+f)}{k}\right) \\
& =\chi^{*}(f) e\left(\frac{m f}{k}\right) \sum_{u=1}^{k / d} e\left(\frac{m u}{k / d}\right) .
\end{aligned}
$$

Since $(m, k)=1$, the last sum vanishes, and consequently,

$$
G(d, f, m, \chi)=0 .
$$

If $d \mid k^{*}$, then on making the substitution $n=u k^{*}+v$ one has

$$
G(d, f, m, \chi)=\sum_{u=1}^{k / k^{*}} \sum_{v=1}^{k^{*}} \chi^{*}\left(u k^{*}+v\right) e\left(\frac{m\left(u k^{*}+v\right)}{k}\right),
$$

where the double sums over $u, v$ are further restricted by the condition $u k^{*}+v \equiv f(\bmod d)$. The restriction $u k^{*}+v \equiv f(\bmod d)$ is equivalent to $v \equiv f(\bmod d)$. Therefore the above quantity can be written as

$$
\sum_{u=1}^{k / k^{*}} e\left(\frac{m u}{k / k^{*}}\right) \sum_{\substack{v=1 \\ v \equiv f(\bmod d)}}^{k^{*}} \chi^{*}(v) e\left(\frac{m v}{k}\right) .
$$

The first sum vanishes unless $k=k^{*}$, hence for $k^{*} \neq k$ one has

$$
G(d, f, m, \chi)=0 .
$$


While for $k^{*}=k$ one obtains

$$
G(d, f, m, \chi)=\sum_{\substack{v=1 \\ v \equiv f(\bmod d)}}^{k^{*}} \chi^{*}(v) e\left(\frac{m v}{k^{*}}\right)=G\left(d, f, m, \chi^{*}\right),
$$

hence by Lemma 4,

$$
|G(d, f, m, \chi)| \leq k^{* 1 / 2} .
$$

We therefore conclude from (3.6)-(3.8) that (3.5) holds for $k=p^{\alpha}$.

General case. We now turn to general $k$. To this end, we first prove that $G(d, f, m, \chi)$ is multiplicative with respect to $k$. Let $k=k_{1} k_{2}$ with $\left(k_{1}, k_{2}\right)=1$. Then for $\chi \bmod k$ there exist a unique couple of characters $\chi_{1} \bmod k_{1}$ and $\chi_{2} \bmod k_{2}$ such that $\chi=\chi_{1} \chi_{2}$. Therefore, on making the substitution $n=k_{2} n_{1}+k_{1} n_{2}$, one has

$$
G(d, f, m, \chi)=\sum_{n_{1}=1}^{k_{1}} \sum_{n_{2}=1}^{k_{2}} \chi_{1} \chi_{2}\left(k_{2} n_{1}+k_{1} n_{2}\right) e\left(\frac{m\left(k_{2} n_{1}+k_{1} n_{2}\right)}{k_{1} k_{2}}\right),
$$

where the double sums are further restricted by

$$
k_{2} n_{1}+k_{1} n_{2} \equiv f(\bmod d) \text {. }
$$

On noting that $d \mid k$, we set $d=d_{1} d_{2}$ with $d_{1} \mid k_{1}$ and $d_{2} \mid k_{2}$. It follows from $\left(k_{1}, k_{2}\right)=1$ that $\left(d_{1}, d_{2}\right)=1$, hence $(3.10)$ is equivalent to

$$
n_{1} \equiv f \bar{k}_{2}\left(\bmod d_{1}\right), \quad n_{2} \equiv f \bar{k}_{1}\left(\bmod d_{2}\right),
$$

where $\bar{k}_{1}$ and $\bar{k}_{2}$ are defined by $k_{1} \bar{k}_{1} \equiv 1\left(\bmod d_{2}\right)$ and $k_{2} \bar{k}_{2} \equiv 1\left(\bmod d_{1}\right)$. Now (3.9) becomes

$$
\begin{aligned}
& G(d, f, m, \chi) \\
= & \sum_{\substack{n_{1}=1 \\
n_{1} \equiv f \bar{k}_{2}\left(\bmod d_{1}\right)}}^{k_{1}} \chi_{1}\left(k_{2} n_{1}\right) e\left(\frac{m n_{1}}{k_{1}}\right) \sum_{\substack{n_{2}=1 \\
n_{2} \equiv f \bar{k}_{1}\left(\bmod d_{2}\right)}}^{k_{2}} \chi_{2}\left(k_{1} n_{2}\right) e\left(\frac{m n_{2}}{k_{2}}\right) \\
= & \chi_{1}\left(k_{2}\right) \chi_{2}\left(k_{1}\right) G\left(d_{1}, f \bar{k}_{2}, m, \chi_{1}\right) G\left(d_{2}, f \bar{k}_{1}, m, \chi_{2}\right) .
\end{aligned}
$$

Now let

$$
k=p_{1}^{\alpha_{1}} p_{2}^{\alpha_{2}} \ldots p_{s}^{\alpha_{s}}
$$

be the canonical decomposition of $k$, where $p_{j}$ stands for primes, and $\alpha_{j}$ positive integers. Accordingly, $k^{*}$ and $d$ can be written as

$$
k^{*}=p_{1}^{\beta_{1}} p_{2}^{\beta_{2}} \ldots p_{s}^{\beta_{s}} \quad \text { and } \quad d=p_{1}^{\gamma_{1}} p_{2}^{\gamma_{2}} \ldots p_{s}^{\gamma_{s}},
$$

where $\beta_{j}$ and $\gamma_{j}$ are integers satisfying $1 \leq \beta_{j} \leq \alpha_{j}$ and $0 \leq \gamma_{j} \leq \alpha_{j}$. It follows that there are primitive characters $\chi_{j}^{*} \bmod p_{j}^{\beta_{j}}, j=1, \ldots, s$, such that $\chi^{*}=\chi_{1}^{*} \chi_{2}^{*} \cdots \chi_{s}^{*}$, and each $\chi_{j}^{*} \bmod p_{j}^{\beta_{j}}$ induces $\chi_{j} \bmod p_{j}^{\alpha_{j}}$. 
Making the substitution $n=n_{1} K_{1}+n_{2} K_{2}+\ldots+n_{s} K_{s}$, where $K_{j}$ is defined by $p_{j}^{\alpha_{j}} K_{j}=k$, one sees that

$$
G(d, f, m, \chi)=\prod_{j=1}^{s} \chi_{j}\left(K_{j}\right) G\left(p_{j}^{\gamma_{j}}, f \bar{K}_{j}, m, \chi_{j}\right)
$$

where $\bar{K}_{j}$ satisfies

$$
\bar{K}_{j} K_{j} \equiv 1\left(\bmod p_{j}^{\gamma_{j}}\right), \quad j=1, \ldots, s .
$$

It follows that

$$
|G(d, f, m, \chi)| \leq \prod_{j=1}^{s}\left|G\left(p_{j}^{\gamma_{j}}, f \bar{K}_{j}, m, \chi_{j}\right)\right|=\prod_{j=1}^{s} p_{j}^{\beta_{j} / 2}=k^{* 1 / 2} .
$$

This completes the proof of the lemma.

Lemma 7. Let $d \mid k$ and $(m, k)=(f, k)=1$. Let also $\chi^{0} \bmod k$ be the principal character. Then for $(d, k / d)>1$,

$$
G\left(d, f, m, \chi^{0}\right)=0
$$

and for $(d, k / d)=1$,

$$
G\left(d, f, m, \chi^{0}\right)=\mu\left(\frac{k}{d}\right) e\left(\frac{f m t}{d}\right),
$$

where $t$ is defined by $t k / d \equiv 1(\bmod d)$.

This is Hilfssatz 2 of Rademacher [R] or Theorem 2.2 of Ayoub [A].

We can now give

Proof of Lemma 4. Let

$$
k=k_{1} k_{2} \quad \text { with } \quad\left(k_{1}, k_{2}\right)=1, \quad k^{*} \mid k_{1}, \quad \text { and } \quad p\left|k_{1} \Rightarrow p\right| k^{*} .
$$

Then for $\chi \bmod k$ there exist a unique couple of characters $\chi_{1} \bmod k_{1}$ and $\chi_{2}^{0} \bmod k_{2}$ such that $\chi=\chi_{1} \chi_{2}^{0}$, where $\chi_{2}^{0} \bmod k_{2}$ is the principal character. On noting that $d \mid k$, we set $d=d_{1} d_{2}$ with $d_{1} \mid k_{1}$ and $d_{2} \mid k_{2}$. It therefore follows from (3.12) that

$$
G(d, f, m, \chi)=\chi_{1}\left(k_{2}\right) \chi_{2}^{0}\left(k_{1}\right) G\left(d_{1}, f \bar{k}_{2}, m, \chi_{1}\right) G\left(d_{2}, f \bar{k}_{1}, m, \chi_{2}^{0}\right) .
$$

The statement of the lemma now follows from Lemmas 6 and 7.

4. A mean-value estimate for exponential sums over primes. Wolke [W] was the first to study the mean-value estimate as in Theorem 3 . He proved that Theorem 3 is true for

$$
1 \leq K=x^{1 / 4}, \quad \theta=\min \left(K^{-4}, L^{-E}\right) .
$$

Actually, Theorem 3 was recently given by the authors in another joint paper [ZL] as an improvement of Wolke's result. Unfortunately, however, there is 
a gap in the proof of [ZL]: the statement " $h$ " $(\beta)>0$ for $1 / 2 \leq \beta \leq 1$ " on p. 365 of [ZL] is not always true. The proof therefore needs corrections.

In this section we prove the following general result, which contains the assertion of Theorem 3. One can see from the proof of Theorem 2 that this general theorem is necessary.

THEOREM 4. Let $z \geq 1$ be arbitrary. For any $A>0$, there exists a constant $E=E(A)>0$ such that if

$$
1 \leq K \leq z^{2 / 3} x^{1 / 3} L^{-E}, \quad \theta=z^{2} K^{-3} L^{-E},
$$

then

$\sum_{q \leq K} \max _{y \leq x} \max _{(a, q)=1} \max _{|\lambda| \leq \theta}\left|\sum_{n \leq y} \Lambda(n) e\left(n\left(\frac{a}{q}+\lambda\right)\right)-\frac{\mu(q)}{\varphi(q)} \sum_{n \leq y} e(n \lambda)\right| \ll z x L^{-A}$.

We need some lemmas to establish this result.

Lemma 8. Suppose that $F(u)$ and $G(u)$ are real functions defined on $[a, b]$, and $G(u)$ and $1 / F^{\prime}(u)$ are monotonic.

(i) If $\left|F^{\prime}(u)\right| \gg m$ and $|G(u)| \ll M$, then

$$
\int_{a}^{b} G(u) e(F(u)) d u \ll M / m .
$$

(ii) If $\left|F^{\prime \prime}(u)\right| \gg r$ and $|G(u)| \ll M$, then

$$
\int_{a}^{b} G(u) e(F(u)) d u \ll M / \sqrt{r} .
$$

For the proof of these results, see Lemmas 3.3 and 3.4 in Titchmarsh $[\mathrm{T}]$.

Lemma 9. Let $N(\sigma, T, \chi)$ be the number of zeros $\varrho=\beta+i \gamma$ of the Dirichlet $L$-function $L(s, \chi)$ in the rectangle $\sigma \leq \beta \leq 1,-T \leq \gamma \leq T$. Suppose $q \leq 1$ and $T \leq 2$. Then, for $1 / 2 \leq \sigma \leq 1$, we have

$$
\sum_{\chi \bmod q} N(\sigma, T, \chi) \ll(q T)^{3(1-\sigma) /(2-\sigma)}(\log q T)^{9} .
$$

This is Theorem 12.1 in Montgomery [Mo].

Lemma 10. Let $a_{n}, n=1,2, \ldots$, be complex numbers and $\chi \bmod q a$ character. Then

$$
\sum_{q \leq Q} \sum_{\chi \bmod }^{*} \int_{q}^{T}\left|\sum_{n \leq N} a_{n} \chi(n) n^{i t}\right|^{2} d t \ll\left(Q^{2} T+N\right) \sum_{n \leq N}\left|a_{n}\right|^{2}
$$

for arbitrary $Q, T_{0}$, and $T$.

For this, see Theorem 7.1 in Montgomery [Mo]. 
Lemma 11. Let $\zeta(s)$ be the Riemann zeta-function, and

$$
F(s)=\sum_{n \leq U} \Lambda(n) / n^{s}, \quad G(s)=\sum_{n \leq U} \mu(n) / n^{s} .
$$

Then

$$
\begin{aligned}
\left(-\frac{\zeta^{\prime}}{\zeta}(s)-F(s)\right)= & G(s)\left(-\zeta^{\prime}(s)\right)-F(s) G(s) \zeta(s) \\
& -(\zeta(s) G(s)-1)\left(-\frac{\zeta^{\prime}}{\zeta}(s)-F(s)\right) .
\end{aligned}
$$

This is Vaughan's identity; for the proof, see [Va].

Now we can, using the idea due to Zhan [Zh], give the proof of Theorem 4.

Proof of Theorem 4. Introducing the Dirichlet characters, the exponential sum under consideration becomes

$$
\begin{aligned}
\sum_{n \leq y} \Lambda(n) e & \left(n\left(\frac{a}{q}+\lambda\right)\right) \\
& =\frac{1}{\varphi(q)} \sum_{\chi \bmod q} \sum_{n \leq y} \Lambda(n) \chi(n) e(n \lambda) \sum_{h=1}^{q} \bar{\chi}(h) e\left(\frac{a h}{q}\right)+O\left(L^{2}\right),
\end{aligned}
$$

and consequently,

$$
\begin{aligned}
& \text { (4.2) } \quad \sum_{q \leq Q} \max _{y \leq x} \max _{|\lambda| \leq \theta} \max _{(a, q)=1}\left|\sum_{n \leq y} \Lambda(n) e\left(n\left(\frac{a}{q}+\lambda\right)\right)-\frac{\mu(q)}{\varphi(q)} \sum_{n \leq y} e(n \lambda)\right| \\
& \ll \sum_{q \leq K} \max _{y \leq x} \max _{|\lambda| \leq \theta} \max _{(a, q)=1} \frac{1}{\varphi(q)} \sum_{\chi \bmod q}\left|G(a, \chi) \sum_{n \leq y} \Lambda(n ; \chi) \chi(n) e(n \lambda)\right|+K L^{2},
\end{aligned}
$$

where $G(a, \chi)$ is defined as in $\S 3$, and

$$
\Lambda(n ; \chi)= \begin{cases}\Lambda(n) & \text { for } \chi \neq \chi^{0} \\ \Lambda(n)-1 & \text { for } \chi=\chi^{0}\end{cases}
$$

To estimate the sums on the right-hand side of (4.2), one notes that if the primitive character $\chi \bmod q$ induces the character $\eta \bmod k$, then $q \mid k$, and $|G(a, q)| \leq q^{1 / 2}$ for $(a, q)=1$. We now combine all contributions made by an individual primitive character, so that the first term on the right-hand side of (4.2) is

$$
\begin{aligned}
& \ll \sum_{q \leq K} \max _{y \leq x} \max _{|\lambda| \leq \theta} \max _{(a, q)=1} \sum_{\substack{k \leq K \\
q \mid k}} \frac{q^{1 / 2}}{\varphi(k)} \sum_{\chi \bmod q}^{*}\left|\sum_{n \leq y} \Lambda(n ; \chi) \chi(n) e(n \lambda)\right| \\
& \ll L \sum_{q \leq K} \max _{y \leq x} \max _{|\lambda| \leq \theta} \frac{q^{1 / 2}}{\varphi(q)} \sum_{\chi \bmod q}^{*}\left|\sum_{n \leq y} \Lambda(n ; \chi) \chi(n) e(n \lambda)\right| .
\end{aligned}
$$


Hence the assertion of the theorem reduces to

$$
S:=\sum_{q \sim D} \max _{y \leq x} \max _{|\lambda| \leq \theta} \sum_{\chi \bmod q}^{*}\left|\sum_{n \sim y} \Lambda(n ; \chi) \chi(n) e(n \lambda)\right| \ll z x D^{1 / 2} L^{-A-3},
$$

with $1 \leq D \leq K$ and $K, \theta$ satisfying (4.1).

The argument leading to (4.3) falls naturally into two cases according as $D$ is small or large. For $D \leq L^{F}$, where $F$ is some positive constant, one uses the classical zero-density estimate and zero-free region for the Dirichlet $L$-functions. While for $L^{F}<D \leq K$, one appeals to contour integration, the large sieve inequality and Vaughan's identity.

CASE $1 . D \leq L^{F}$, where $F$ is a positive constant to be specified later in terms of $A$. In this case, it suffices to prove that

$$
\Sigma:=\sum_{n \sim y} \Lambda(n ; \chi) \chi(n) e(n \lambda) \ll z x L^{-2 F-A-3}
$$

for $y \leq x,|\lambda| \leq \theta$ and any primitive character $\chi \bmod d$.

To estimate $\Sigma$, one appeals to the Siegel-Walfisz theorem ([D], §19):

$$
\sum_{n \sim u} \Lambda(n ; \chi) \chi(n) e(n \lambda)=-\sum_{|\gamma| \leq T} \frac{u^{\varrho}}{\varrho}+b(\chi)+O\left(\frac{u(\log u q T)^{2}}{T}\right)
$$

where $\varrho=\beta+i \gamma$ denotes nontrivial zeros of $L(s, \chi), b(\chi)$ is a constant depending on $\chi$, and $T \geq 2$ is a parameter. Applying partial summation, we have

$$
\begin{aligned}
\Sigma & =\int_{y / 2}^{y} e(\lambda u) d\left(\sum_{n \leq u} \Lambda(n ; \chi) \chi(n)\right) \\
& =\sum_{|\gamma| \leq T} \int_{y / 2}^{y} u^{\varrho-1} e(\lambda u) d u+O\left((1+|\lambda| x) \frac{x L^{2}}{T}\right) .
\end{aligned}
$$

Take $T=x^{2}$, so that the $O$-term is acceptable in (4.4). Since, for $u \sim y$,

$$
\frac{d}{d u}\left(\lambda u+\frac{\gamma}{2 \pi} \log u\right)=\lambda+\frac{\gamma}{2 \pi u} \gg \frac{\min _{u \sim y}|\gamma+2 \pi \lambda u|}{y},
$$

and

$$
\frac{d^{2}}{d u^{2}}\left(\lambda u+\frac{\gamma}{2 \pi} \log u\right)=-\frac{\gamma}{2 \pi u^{2}} \gg \frac{|\gamma|}{y^{2}},
$$

we deduce from Lemma 8 that the integral on the right-hand side of (4.5) is

$$
\int_{y / 2}^{y} u^{\beta-1} e\left(\lambda u+\frac{\gamma}{2 \pi} \log u\right) d u \ll \min \left(\frac{y^{\beta}}{\sqrt{|\gamma|+1}}, \frac{y^{\beta}}{\min _{u \sim y}|\gamma+2 \pi \lambda u|}\right) .
$$


Let $T_{0}=4 \pi \theta x$, so that for $T_{0}<|\gamma| \leq x^{2}$ and $u \sim y$,

$$
|\gamma+2 \pi \lambda u| \geq|\gamma|-2 \pi \theta u \geq|\gamma| / 2 \text {. }
$$

Then (4.5) becomes

$$
\begin{aligned}
\Sigma & \ll \sum_{|\gamma| \leq x^{2}} \min \left(\frac{y^{\beta}}{\sqrt{|\gamma|+1}}, \frac{y^{\beta}}{\min _{u \sim y}|\gamma+2 \pi \lambda u|}\right)+O\left(x L^{-2 F-A-3}\right) \\
& \ll \sum_{|\gamma| \leq T_{0}} \frac{x^{\beta}}{\sqrt{|\gamma|+1}}+\sum_{T_{0}<|\gamma| \leq x^{2}} \frac{x^{\beta}}{|\gamma|}+O\left(x L^{-2 F-A-3}\right) .
\end{aligned}
$$

It is well known that for any $\chi \bmod q$ there is a constant $c_{1}>0$ such that $L(s, \chi)$ has no zero in the region

$$
\sigma \geq 1-\frac{c_{1}}{\log q+\log ^{4 / 5}(|t|+2)}
$$

except the possible Siegel zero. But the Siegel zero does not exist in the present situation, since $q \leq L^{F}$. Therefore, one has

$$
x^{\beta-1} \ll \exp \left\{-\frac{c_{1} \log x}{\log q+\log ^{4 / 5} T}\right\} \ll \exp \left(-c_{2} L^{1 / 5}\right),
$$

for some constant $c_{2}>0$. Hence the second sum on the right-hand side of (4.6) is acceptable.

To deal with the first term, one notes that

$$
\sum_{|\gamma| \leq T_{0}} \frac{x^{\beta}}{\sqrt{|\gamma|+1}} \ll x L \max _{T_{1} \sim T_{0}} T_{1}^{-1 / 2} \sum_{|\gamma| \leq T_{1}} x^{\beta-1},
$$

which is, on applying Lemma 9 ,

$$
\begin{aligned}
& \ll x L \max _{T_{1} \leq T_{0}} T_{1}^{-1 / 2}\left(\log q T_{1}\right)^{9} \max _{1 / 2 \leq \sigma \leq 1}\left(q T_{1}\right)^{(3-3 \sigma) /(2-\sigma)} x^{\sigma-1} \\
& \ll x L^{F+11} \max _{T_{1} \leq T_{0}} \max _{1 / 2 \leq \sigma \leq 1} \exp \left\{-(1-\sigma) L+\left(\frac{3-3 \sigma}{2-\sigma}-\frac{1}{2}\right) \log T_{1}\right\} \\
& =: x L^{F+11} \max _{T_{1} \leq T_{0}} \max _{1 / 2 \leq \sigma \leq 1} f\left(T_{1}, \sigma\right),
\end{aligned}
$$

say. Therefore, in view of (4.6), the estimate (4.4) reduces to

$$
\max _{T_{1} \leq T_{0}} \max _{1 / 2 \leq \sigma \leq 1} f\left(T_{1}, \sigma\right) \ll z L^{-3 F-A-20} .
$$

Suppose first $4 / 5 \leq \sigma \leq 1$, so that

$$
\frac{3-3 \sigma}{2-\sigma} \leq \frac{1}{2}
$$

It follows from (4.7) that

$$
\max _{T_{1} \leq T_{0}} \max _{4 / 5 \leq \sigma \leq 1} f\left(T_{1}, \sigma\right) \ll \max _{4 / 5 \leq \sigma \leq 1} \exp \{-(1-\sigma) L\} \ll \exp \left\{-c_{2} L^{1 / 5}\right\}
$$


which is acceptable in (4.8). Now we turn to $3 / 5 \leq \sigma \leq 4 / 5$, which ensures that

$$
\frac{3-3 \sigma}{2-\sigma} \geq \frac{1}{2}
$$

On noting that $\log T_{1} \leq L+O(1)$, and

$$
\max _{3 / 5 \leq \sigma \leq 4 / 5}\left\{-\frac{\sigma(\sigma-1 / 2)}{2-\sigma}\right\}=-\frac{3}{70},
$$

one deduces that

$$
\begin{aligned}
\max _{T_{1} \leq T_{0}} \max _{3 / 5 \leq \sigma \leq 4 / 5} f\left(T_{1}, \sigma\right) & \ll \max _{3 / 5 \leq \sigma \leq 4 / 5} \exp \left\{-(1-\sigma) L+\left(\frac{3-3 \sigma}{2-\sigma}-\frac{1}{2}\right) L\right\} \\
& =\max _{3 / 5 \leq \sigma \leq 4 / 5} \exp \left\{-\frac{\sigma(\sigma-1 / 2)}{2-\sigma} L\right\} \\
& \ll x^{-3 / 70},
\end{aligned}
$$

and this is also acceptable in (4.8). Finally, we consider $1 / 2 \leq \sigma \leq 3 / 5$. Now we have

$$
\frac{6}{7} \leq \frac{3-3 \sigma}{2-\sigma}-\frac{1}{2}
$$

and consequently,

$$
\begin{aligned}
& \max _{T_{1} \leq T_{0} 0} \max _{1 / 2 \leq \sigma \leq 3 / 5} f\left(T_{1}, \sigma\right) \\
& \ll \max _{T_{1} \leq T_{0}} \max _{1 / 2 \leq \sigma \leq 3 / 5} \exp \left\{-(1-\sigma) L+\left(\frac{3-3 \sigma}{2-\sigma}-\frac{1}{2}\right) \log x\right\} \\
& \quad \times \exp \left\{-\left(\frac{3-3 \sigma}{2-\sigma}-\frac{1}{2}\right) \log \frac{x}{T_{1}}\right\} .
\end{aligned}
$$

Since $T_{1} \ll T_{0} \ll \theta x \ll x L^{-E}$, the above quantity is

$$
\begin{aligned}
& \ll \max _{1 / 2 \leq \sigma \leq 3 / 5} \exp \left\{-\frac{\sigma(\sigma-1 / 2)}{2-\sigma} L\right\} \exp \left\{-\frac{6}{7} E \log \log x\right\} \\
& \ll L^{-6 E / 7},
\end{aligned}
$$

which is acceptable in (4.8) if $E \geq 6 F+2 A+28$.

Combining (4.9)-(4.11) we get (4.8), hence (4.4). This proves (4.3) in Case 1.

CASE 2. $L^{F}<D \leq K$, where $F$ is a constant to be specified in the following argument. In this case, we use Vaughan's identity to establish (4.3). 
Estimation of the sum of type $I$. We first show that

$$
\begin{aligned}
\Sigma^{\prime} & :=\sum_{d \sim D} \max _{y \leq x} \max _{|\lambda| \leq \theta} \sum_{\chi \bmod d}^{*}\left|\sum_{\substack{m n \sim y \\
m \sim M \\
n \sim N}} a(m) b(n) \chi(m n) e(m n \lambda)\right| \\
& \ll z x D^{1 / 2} L^{-A-7}
\end{aligned}
$$

holds for $a(m) \ll d(m)$ and $b(n) \ll d(n)$ with $m \sim M, n \sim N$ and

$$
x \ll M N \ll x, \quad M, N \leq x D^{-1} L^{-2 A-20} .
$$

Let

$$
f_{1}(s, \chi)=\sum_{m \sim M} \frac{a(m) \chi(m)}{m^{s}} \text { and } f_{2}(s, \chi)=\sum_{n \sim N} \frac{b(n) \chi(n)}{n^{s}},
$$

where $s=\sigma+i t$ is a complex variable. Then one sees that

$$
f_{1}(s, \chi) f_{2}(s, \chi) \ll M^{1-\sigma} N^{1-\sigma} \ll x^{1-\sigma}
$$

uniformly for $-2 \leq \sigma \leq 2$. Applying Perron's summation formula (see e.g.

Lemma 3.12 in $[\mathrm{T}]$ ) and then shifting the contour to the left, one gets

$$
\begin{aligned}
& \sum_{\substack{m n \leq u \\
m \sim M \\
n \sim N}} a(m) b(n) \chi(m) \chi(n) \\
& =\frac{1}{2 \pi i} \int_{1+\varepsilon-i x^{2}}^{1+\varepsilon+i x^{2}} f_{1}(s, \chi) f_{2}(s, \chi) \frac{u^{s}}{s} d s+O(L) \\
& =\frac{1}{2 \pi i}\left\{\int_{1+\varepsilon-i x^{2}}^{1 / 2-i x^{2}}+\int_{1 / 2-i x^{2}}^{1 / 2+i x^{2}}+\int_{1 / 2+i x^{2}}^{1+\varepsilon+i x^{2}}\right\} f_{1}(s, \chi) f_{2}(s, \chi) \frac{u^{s}}{s} d s+O(L) .
\end{aligned}
$$

By (4.14), the integrals on the horizontal parts are clearly $O(L)$. Therefore,

$$
\begin{aligned}
& \sum_{\substack{m n \leq u \\
m \sim M \\
n \sim N}} a(m) b(n) \chi(m) \chi(n) \\
& \quad=\frac{1}{2 \pi} \int_{-x^{2}}^{x^{2}} f_{1}\left(\frac{1}{2}+i t, \chi\right) f_{2}\left(\frac{1}{2}+i t, \chi\right) \frac{u^{1 / 2+i t}}{1 / 2+i t} d t+O(L) .
\end{aligned}
$$

Now, by partial summation, the inner sum of $\Sigma^{\prime}$ is

$$
\begin{aligned}
\int_{y / 2}^{y} e(\lambda u) d\left\{\sum_{\substack{m n \leq u \\
m \sim M \\
n \sim N}} a(m) b(n) \chi(m) \chi(n)\right\} \\
=\frac{1}{2 \pi} \int_{-x^{2}}^{x^{2}} f_{1}\left(\frac{1}{2}+i t, \chi\right) f_{2}\left(\frac{1}{2}+i t, \chi\right) \frac{1}{1 / 2+i t} \int_{y / 2}^{y} u^{1 / 2+i t} e(\lambda u) d u d t \\
+O((1+|\lambda| x) L)
\end{aligned}
$$




$$
\begin{aligned}
= & \frac{1}{2 \pi} \int_{-x^{2}}^{x^{2}} f_{1}\left(\frac{1}{2}+i t, \chi\right) f_{2}\left(\frac{1}{2}+i t, \chi\right) \\
& \times \frac{1}{1 / 2+i t} \int_{y / 2}^{y} u^{-1 / 2} e\left(\lambda+\frac{t}{2 \pi} \log u\right) d u d t+O(\theta x L),
\end{aligned}
$$

which, by the argument leading to (4.6), is estimated as

$$
\begin{aligned}
& \ll x^{1 / 2} \int_{|t| \leq T_{0}}\left|f_{1}\left(\frac{1}{2}+i t, \chi\right) f_{2}\left(\frac{1}{2}+i t, \chi\right)\right| \frac{d t}{\sqrt{|t|+1}} \\
& +x^{1 / 2} \int_{T_{0}<|t| \leq x^{2}}\left|f_{1}\left(\frac{1}{2}+i t, \chi\right) f_{2}\left(\frac{1}{2}+i t, \chi\right)\right| \frac{d t}{|t|}+O(\theta x L) .
\end{aligned}
$$

It therefore suffices to show

$$
\begin{aligned}
\sum_{d \sim D} \sum_{\chi \bmod }^{*} \int_{T_{2} / 2}^{T_{2}}\left|f_{1}\left(\frac{1}{2}+i t, \chi\right) f_{2}\left(\frac{1}{2}+i t, \chi\right)\right| d t \\
\\
\ll z x^{1 / 2} D^{1 / 2} T_{2}^{1 / 2} L^{-A-8}
\end{aligned}
$$

for $1 \leq T_{2} \leq T_{0}$, and

$$
\begin{aligned}
\sum_{d \sim D} \sum_{\chi \bmod }^{*} \int_{d T_{3} / 2}^{T_{3}}\left|f_{1}\left(\frac{1}{2}+i t, \chi\right) f_{2}\left(\frac{1}{2}+i t, \chi\right)\right| d t & \\
& \ll z x^{1 / 2} D^{1 / 2} T_{3} L^{-A-8}
\end{aligned}
$$

for $T_{0} \leq T_{3} \leq x^{2}$.

The left-hand side of (4.15) is, by Cauchy's inequality and Lemma 10,

$$
\begin{aligned}
& \ll\left\{\sum_{d \sim D} \sum_{\chi \bmod }^{*} \int_{d T_{2} / 2}^{T_{2}}\left|f_{1}\left(\frac{1}{2}+i t, \chi\right)\right|^{2} d t\right\}^{1 / 2} \\
& \times\left\{\sum_{d \sim D} \sum_{\chi \bmod }^{*} \int_{d T_{2} / 2}^{T_{2}}\left|f_{2}\left(\frac{1}{2}+i t, \chi\right)\right|^{2} d t\right\}^{1 / 2} \\
& \ll\left(D^{2} T_{2}+M\right)^{1 / 2}\left(D^{2} T_{2}+N\right)^{1 / 2} L \\
& \ll\left.\ll D^{2} T_{2}+D T_{2}^{1 / 2}\left(M^{1 / 2}+N^{1 / 2}\right)+M^{1 / 2} N^{1 / 2}\right\} L \\
& \ll z x^{1 / 2} D^{1 / 2} T_{2}^{1 / 2} L^{-A-8}
\end{aligned}
$$

if $F \geq 2 A+20$ and $E \geq 2 A+20$. This yields (4.15).

A similar argument gives (4.16). This proves (4.12) subject to (4.13). 
Estimation of the sum of type II. Next we prove that

$$
\begin{aligned}
\Sigma^{\prime \prime} & :=\sum_{d \sim D} \max _{y \leq x} \max _{|\lambda| \leq \theta} \sum_{\chi \bmod d}^{*}\left|\sum_{\substack{m n \sim y \\
m \sim M \\
n \sim N}} b(n) \chi(m n) e(m n \lambda)\right| \\
& \ll z x D^{1 / 2} L^{-A-7}
\end{aligned}
$$

holds for $b(n) \ll d(n)$ with $n \sim N$ and $M, N$ satisfying

$$
x \ll M N \ll x, \quad M \geq D L^{2 A+20} .
$$

Arguing as before, one sees that it suffices to show (4.15) and (4.16) subject to (4.19). Here $f_{1}(s, \chi), f_{2}(s, \chi)$ are the same as before except that $a(m)=1$ in the definition of $f_{1}(s, \chi)$. Since now $M$ is large according to (4.19), the above approach to attack the mean value of $f_{1}(s, \chi)$ does not work any more; one therefore needs to treat $f_{1}(s, \chi)$ differently.

Let $w=u+i v$ be a complex variable. Then, applying Perron's formula and then shifting the line of integration as before, one gets

$$
\begin{aligned}
f_{1}\left(\frac{1}{2}+i t, \chi\right) & =\frac{1}{2 \pi i} \int_{1+\varepsilon-i x^{2}}^{1+\varepsilon+i x^{2}} L\left(\frac{1}{2}+i t+w, \chi\right) \frac{M^{w}-(M / 2)^{w}}{w} d w+O(L) \\
& =\frac{1}{2 \pi} \int_{-x^{2}}^{x^{2}} L\left(\frac{1}{2}+i t+i v, \chi\right) \frac{M^{i v}-(M / 2)^{i v}}{i v} d v+O(L) \\
& \ll \int_{-x^{2}}^{x^{2}} \frac{1}{|v|+1}\left|L\left(\frac{1}{2}+i t+i v, \chi\right)\right| d v+O(L) .
\end{aligned}
$$

Consequently, by Cauchy's inequality,

$$
\begin{aligned}
& \left|f_{1}\left(\frac{1}{2}+i t, \chi\right)\right|^{2} \\
& \quad \ll\left\{\int_{-x^{2}}^{x^{2}} \frac{1}{|v|+1} d v\right\}\left\{\int_{-x^{2}}^{x^{2}} \frac{1}{|v|+1}\left|L\left(\frac{1}{2}+i t+i v, \chi\right)\right|^{2} d v\right\}+L^{2} \\
& \quad \ll L \int_{-x^{2}}^{x^{2}} \frac{1}{|v|+1}\left|L\left(\frac{1}{2}+i t+i v, \chi\right)\right|^{2} d v+L^{2} .
\end{aligned}
$$

It follows that

$$
\begin{aligned}
& \sum_{d \sim D} \sum_{\chi \bmod }^{*} \int_{d T_{2} / 2}^{T_{2}}\left|f_{1}\left(\frac{1}{2}+i t, \chi\right)\right|^{2} d t \\
& \ll L \max _{T_{4} \leq x^{2}} \frac{1}{T_{4}} \sum_{d \sim D} \sum_{\chi \bmod d}^{*} \int_{T_{2} / 2}^{T_{2}} \int_{T_{4} / 2}^{T_{4}}\left|L\left(\frac{1}{2}+i t+i v, \chi\right)\right|^{2} d v d t+D^{2} T_{2} L^{2}
\end{aligned}
$$




$$
\begin{aligned}
\ll & L \max _{T_{2}<T_{4} \leq x^{2}} \frac{1}{T_{4}} \int_{T_{2} / 2}^{T_{2}}\left\{\sum_{d \sim D} \sum_{\chi \bmod d}^{*} \int_{T_{4} / 2+t}^{T_{4}+t}\left|L\left(\frac{1}{2}+i \tau, \chi\right)\right|^{2} d \tau\right\} d t \\
& +L \max _{T_{4} \leq T_{2}} \frac{1}{T_{4}} \int_{T_{4} / 2}^{T_{4}}\left\{\sum_{d \sim D} \sum_{\chi \bmod d}^{*} \int_{T_{2} / 2+v}^{T_{2}+v}\left|L\left(\frac{1}{2}+i \tau, \chi\right)\right|^{2} d \tau\right\} d v \\
& +D^{2} T_{2} L^{2} .
\end{aligned}
$$

Applying the classical estimate

$$
\sum_{\chi \bmod q}^{*} \int_{0}^{T}\left|L\left(\frac{1}{2}+i t, \chi\right)\right|^{2} d t \ll q T(\log q T)^{2},
$$

the quantity above is

$$
\ll D^{2} T_{2} L^{3}+D^{2} T_{2} L^{3}+D^{2} T_{2} L^{2} \ll D^{2} T_{2} L^{3} .
$$

Hence by the argument leading to (4.17), one has

$$
\begin{aligned}
& \sum_{\substack{m n \leq u \\
m \sim M \\
n \sim N}} a(m) b(n) \chi(m) \chi(n) \ll\left\{\sum_{d \sim D} \sum_{\chi \bmod }^{*} \int_{T_{2} / 2}^{T_{2}}\left|f_{1}\left(\frac{1}{2}+i t, \chi\right)\right|^{2} d t\right\}^{1 / 2} \\
& \times\left\{\sum_{d \sim D} \sum_{\chi \bmod }^{*} \int_{d T_{2} / 2}^{T_{2}}\left|f_{2}\left(\frac{1}{2}+i t, \chi\right)\right|^{2} d t\right\}^{1 / 2} \\
& \ll\left(D^{2} T_{2} L^{3}\right)^{1 / 2}\left(D^{2} T_{2}+N\right)^{1 / 2} L^{1 / 2} \\
& \ll\left\{D^{2} T_{2}+D T_{2}^{1 / 2} N^{1 / 2}\right\} L^{2} \ll D^{1 / 2} x^{1 / 2} T_{2}^{1 / 2} L^{-A-8}
\end{aligned}
$$

if $E \geq 2 A+20$. This proves (4.15) under the condition of (4.19).

A similar argument gives (4.16). This completes the proof of (4.18) subject to (4.19).

Application of Vaughan's identity. By Lemma 11, one sees that the inner sum of $S$ in (4.3) is equal to

$$
\sum_{n \sim y} \Lambda(n) \chi(m n) e(m n \lambda)=S_{1}-S_{2}-S_{3},
$$

where

$$
\begin{aligned}
S_{1} & =\sum_{\substack{m n \sim y \\
m \leq U}} \mu(m)(\log n) \chi(m n) e(m n \lambda), \\
S_{2} & =\sum_{\substack{m n \sim y \\
m \leq U^{2}}} a(m) \chi(m n) e(m n \lambda)
\end{aligned}
$$




$$
S_{3}=\sum_{\substack{m n \sim y \\ m>U \\ n>U}} a(m)(\log n) \chi(m n) e(m n \lambda),
$$

and $a(m) \leq d(m)$. Therefore,

$$
\begin{aligned}
S= & \sum_{q \sim D} \max _{y \leq x} \max _{|\lambda \bmod q|} \sum_{\chi \bmod q}^{*}\left|S_{1}\right|+\sum_{q \sim D} \max _{y \leq x} \max _{|\lambda \bmod q|} \sum_{\chi \bmod q}^{*}\left|S_{2}\right| \\
& +\sum_{q \sim D} \max _{y \leq x} \max _{|\lambda \bmod q|} \sum_{\chi \bmod q}^{*}\left|S_{3}\right| .
\end{aligned}
$$

Taking $U=D L^{2 A+20}$ in (4.20) and $E \geq 2 A+20$ in (4.1), we have

$$
U^{2}=D^{2} L^{4 A+40} \leq x D^{-1} L^{-2 A-20} .
$$

Hence each of the three terms on the right-hand side of (4.20) can be divided into $O\left(L^{4}\right)$ sums of the form $\Sigma^{\prime}$ or $\Sigma^{\prime \prime}$. Now, in view of the choice of $E$, (4.12) and (4.18) are both valid, from which the desired result (4.3) for Case 2 follows in the standard way. This completes the proof of the theorem.

5. Preparation for the major arcs. Let $q, r$ be positive integers and

$$
(q, r)=h \text {. }
$$

For $(a, q)=1$ and $(b, r)=1$, define

$$
f(r, q, a, b)= \begin{cases}\frac{\mu(q / h)}{\varphi(r q / h)} e\left(\frac{a b t}{h}\right) & \text { if }(q / h, h)=1, t q / h \equiv 1(\bmod h), \\ 0 & \text { if }(q / h, h)>1 .\end{cases}
$$

And for $S(\alpha ; r, b)$ defined by $(2.4)$, let

$$
\begin{aligned}
E(r, q, a, b, \lambda) & =S\left(\frac{a}{q}+\lambda ; r, b\right)-f(r, q, a, b) \sum_{n \leq N} e(n \lambda), \\
E^{*}(r, q) & =\max _{(a, q)=1} \max _{(b, r)=1|\lambda| \leq 1 /(q Q)} \max _{\mid}|E(r, q, a, b, \lambda)| .
\end{aligned}
$$

The purpose of this section is to establish the following mean-value estimate, which plays an important role in proving (2.5), hence Theorem 2.

Lemma 12. Let $R, P$ and $Q$ be defined as in (2.1) and (2.2), while $f$, $E$ and $E^{*}$ as in (5.2), (5.3) and (5.4). Then for any $A>0$, there exists a constant $C>0$ such that

$$
\sum_{r \sim R} \sum_{q \leq P} E^{*}(r, q) \ll N L^{-A}
$$

This estimate depends on Lemma 13 below, Lemma 3 of $\S 3$, and (4.3) of $\S 4$ which implies Theorem 4. 
Lemma 13. Let $r$ and $q$ be positive integers, and $h, h_{1}, h_{2}$ be defined as in (2.10), (2.11) and (2.12) respectively so that (2.13) holds. Then for fixed positive integers $r^{*}, q^{*}$, one has

$$
\sum_{\substack{r \leq N_{1} \\ r^{*} \mid r / h_{1}}} \sum_{\substack{q \leq N_{2} \\ q^{*} \mid q / h_{2}}} \frac{1}{\varphi\left(r / h_{1}\right) \varphi\left(q / h_{2}\right)} \ll \frac{d\left(r^{*}\right)}{r^{*} q^{*}} \log ^{3} N_{1} \log ^{2} N_{2} .
$$

P ro of. Since $n \ll \varphi(n) \log n$, one has

$$
\sum_{\substack{r \leq N_{1} \\ r^{*} \mid r / h_{1}}} \sum_{\substack{q \leq N_{2} \\ q^{*} \mid q / h_{2}}} \frac{1}{\varphi\left(r / h_{1}\right) \varphi\left(q / h_{2}\right)} \ll \log N_{1} \log N_{2} \sum_{\substack{r \leq N_{1} \\ r^{*} \mid r / h_{1}}} \sum_{\substack{q \leq N_{2} \\ q^{*} \mid q / h_{2}}} \frac{h_{1}}{r} \cdot \frac{h_{2}}{q} .
$$

For a fixed pair $r, h_{1}$, we set $j_{1}=r / h_{1}$. To estimate the sums on the righthand side, one needs the number of pairs $q, h_{2}$ such that the quotients $q / h_{2}$ assume the same value $j_{2}$. Since $h_{2}$ of these pairs must satisfy $h_{2} \mid r / h_{1}$, the required number is obviously $\leq d\left(r / h_{1}\right)$, where $d(n)$ is the divisor function. Hence the double sum under consideration is

$$
\begin{aligned}
\sum_{\substack{r \leq N_{1} \\
r^{*} \mid r / h_{1}}} \sum_{\substack{q \leq N_{2} \\
q^{*} \mid q / h_{2}}} \frac{h_{1} h_{2}}{r q} & \leq \sum_{\substack{j_{1} \leq N_{1} \\
r^{*} \mid j_{1}}} \sum_{\substack{j_{2} \leq N_{2} \\
\left(j_{2}, j_{1}\right)=1 \\
q^{*} \mid j_{2}}} \frac{d\left(j_{1}\right)}{j_{1} j_{2}} \leq \frac{d\left(r^{*}\right)}{r^{*} q^{*}} \sum_{j_{1} \leq N_{1}} \frac{d\left(j_{1}\right)}{j_{1}} \sum_{j_{2} \leq N_{2}} \frac{1}{j_{2}} \\
& \ll \frac{d\left(r^{*}\right)}{r^{*} q^{*}} \log ^{2} N_{1} \log N_{2} .
\end{aligned}
$$

This proves the lemma.

We can now establish the main result of this section.

Proof of Lemma 12. By Lemma 2 we have

$$
\begin{aligned}
S\left(\frac{a}{q}+\right. & \lambda ; r, b) \\
= & \frac{1}{\varphi\left(r / h_{1}\right) \varphi\left(q / h_{2}\right)} \sum_{\xi \bmod r / h_{1}} \bar{\xi}(b) \sum_{\eta \bmod q / h_{2}} G(h, b, a, \bar{\eta}, q) \\
& \times \sum_{n \leq N} \xi \eta(n) \Lambda(n) e(n \lambda)+O\left(L^{2}\right) \\
= & I+J+K+O\left(L^{2}\right),
\end{aligned}
$$

say, where $I, J$ and $K$ are the sums corresponding to

(i) $\xi=\xi^{0} \bmod r / h_{1}, \eta=\eta^{0} \bmod q / h_{2}$,

(j) $\xi=\xi^{0} \bmod r / h_{1}, \eta \neq \eta^{0} \bmod q / h_{2}$,

(k) $\xi \neq \xi^{0} \bmod r / h_{1}$

respectively. 
It is easily seen that

$$
\begin{aligned}
I= & \frac{1}{\varphi\left(r / h_{1}\right) \varphi\left(q / h_{2}\right)} \sum_{\eta=\eta^{0} \bmod q / h_{2}} G(h, b, a, \bar{\eta}, q) \sum_{\substack{n \leq N \\
\chi=\chi^{0} \bmod r q / h}} \chi(n) \Lambda(n) e(n \lambda) \\
= & \frac{1}{\varphi(r q / h)} G\left(h, b, a, \eta_{q / h_{2}}^{0}\right) \\
& \times\left\{\sum_{n \leq N} e(n \lambda)+\sum_{\substack{n \leq N \\
\chi=\chi^{0} \bmod r q / h}} \chi(n)(\Lambda(n)-1) e(n \lambda)+O\left(\frac{L^{2}}{\varphi(r q / h)}\right)\right\} \\
= & f(r, q, a, b) \sum_{n \leq N} e(n \lambda)+O\left(\frac{1}{\varphi(r q / h)} \sum_{n \leq N}(\Lambda(n)-1) e(n \lambda)\right)+O\left(L^{2}\right),
\end{aligned}
$$

where we have used Lemma 5 and (5.2). Taking maxima ever $\lambda, b$ and $a$, and then summing over $q$ and $r$, one gets

$$
\begin{aligned}
\sum_{r \sim R} & \sum_{q \leq P} \max _{(a, q)=1} \max _{(b, r)=1} \max _{|\lambda| \leq 1 /(q Q)}\left|I-f(r, q, a, b) \sum_{n \leq N} e(n \lambda)\right| \\
& \ll \sum_{r \sim R} \frac{1}{\varphi(r)} \sum_{q \leq P} \max _{|\lambda| \leq 1 /(q Q)}\left|\sum_{n \leq N}(\Lambda(n)-1) e(n \lambda)\right|+R P L^{2} \\
& \ll L \sum_{k \leq P} \max _{|\lambda| \leq 1 /(k Q)}\left|\sum_{n \leq N}(\Lambda(n)-1) e(n \lambda)\right|+R P L^{2} .
\end{aligned}
$$

We proceed to estimate $J$. One sees that

$$
\begin{aligned}
J & =\frac{1}{\varphi\left(r / h_{1}\right) \varphi\left(q / h_{2}\right)} \sum_{\eta \bmod q / h_{2}} G(h, b, a, \bar{\eta}, q) \sum_{n \leq N} \xi^{0} \eta(n) \Lambda(n) e(n \lambda) \\
& =\frac{1}{\varphi\left(r / h_{1}\right) \varphi\left(q / h_{2}\right)} \sum_{\eta \bmod q / h_{2}} G(h, b, a, \bar{\eta}, q) \sum_{n \leq N} \eta(n) \Lambda(n) e(n \lambda)+O\left(L^{2}\right) .
\end{aligned}
$$

Consequently, one has

$$
\begin{aligned}
& \sum_{r \sim R} \sum_{q \leq P} \max _{(a, q)=1} \max _{(b, r)=1} \max _{|\lambda| \leq 1 /(q Q)}|J| \\
& \ll \sum_{r \sim R} \frac{1}{\varphi\left(r / h_{1}\right)} \sum_{q \leq P} \frac{1}{\varphi\left(q / h_{2}\right)} \\
& \quad \times \max _{|\lambda| \leq 1 /(q Q)} \sum_{\eta \bmod q / h_{2}}|G(h, b, a, \bar{\eta}, q)|\left|\sum_{n \leq N} \eta(n) \Lambda(n, \eta) e(n \lambda)\right|+P^{3 / 2} L^{3} .
\end{aligned}
$$

To estimate the sums on the right-hand side above, one appeals to Lemma 3 , which ensures that if a primitive character $\chi \bmod k$ induces a character $\bar{\eta} \bmod q / h_{2}$, then $k \mid q / h_{2}$ and $|G(h, b, a, \bar{\eta}, q)| \leq k^{1 / 2}$. We now combine all 
contributions made by an individual primitive character, which gives

$$
\begin{aligned}
& \sum_{r \sim R} \sum_{q \leq P} \max _{(a, q)=1} \max _{(b, r)=1} \max _{|\lambda| \leq 1 /(q Q)}|J| \\
& \ll \sum_{k \leq P}\left\{\sum_{r \sim R} \frac{1}{\varphi\left(r / h_{1}\right)} \sum_{\substack{q \leq P \\
k \mid q / h_{2}}} \frac{k^{1 / 2}}{\varphi\left(q / h_{2}\right)}\right\} \\
& \quad \times \max _{|\lambda| \leq 1 /(k Q)} \sum_{\chi \bmod k}^{*}\left|\sum_{n \leq N} \chi(n) \Lambda(n ; \chi) e(n \lambda)\right|+P^{3 / 2} L^{3} \\
& \ll L^{5} \sum_{k \leq P} \frac{1}{k^{1 / 2}} \max _{|\lambda| \leq 1 /(k Q)} \sum_{\chi \bmod k}^{*}\left|\sum_{n \leq N} \chi(n) \Lambda(n ; \chi) e(n \lambda)\right|+P^{3 / 2} L^{3},
\end{aligned}
$$

where we have used Lemma 13 to estimate the sums in braces.

We now turn to $K$. One has by the definition of $K$,

$$
\begin{aligned}
K= & \frac{1}{\varphi\left(r / h_{1}\right) \varphi\left(q / h_{2}\right)} \sum_{\substack{\xi \bmod r \\
\xi \neq \xi^{0}}} \sum_{\eta \bmod q / h} G(h, b, a, \bar{\eta}, q) \sum_{n \leq N} \xi \eta(n) \Lambda(n) e(n \lambda) \\
\ll & \frac{1}{\varphi\left(r / h_{1}\right) \varphi\left(q / h_{2}\right)} \\
& \times \sum_{\substack{\xi \bmod r / h_{1} \\
\xi \neq \xi^{0}}} \sum_{\eta \bmod q / h_{2}}|G(h, b, a, \bar{\eta}, q)|\left|\sum_{n \leq N} \xi \eta(n) \Lambda(n) e(n \lambda)\right| .
\end{aligned}
$$

Working analogously to the argument above, one sees that

$$
\begin{aligned}
\sum_{r \sim R} \sum_{q \leq P} \max _{(a, q)=1} \max _{(b, r)=1} \max _{|\lambda| \leq 1 /(q Q)}|K| & \ll \sum_{k_{1} \leq 2 R} \sum_{\substack{k_{2} \leq P \\
\left(k_{1}, k_{2}\right)=1}}\left\{\sum_{\substack{r \sim R \\
k_{1} \mid r / h_{1}}} \frac{1}{\varphi\left(r / h_{1}\right)} \sum_{\substack{q \leq P \\
k_{2} \mid q / h_{2}}} \frac{k_{2}^{1 / 2}}{\varphi\left(q / h_{2}\right)}\right\} \\
& \times \max _{|\lambda| \leq 1 /\left(k_{2} Q\right)} \sum_{\substack{\chi_{1} \bmod k_{1} \\
\chi_{1} \neq \chi_{1}^{0}}}^{*} \sum_{\chi_{2} \bmod k_{2}}^{*}\left|\sum_{n \leq N} \chi_{1} \chi_{2}(n) \Lambda(n) e(n \lambda)\right| .
\end{aligned}
$$

By Lemma 13, the quantity in braces is

$$
\ll \frac{d\left(k_{1}\right) k_{2}^{1 / 2}}{k_{1} k_{2}} L^{5} \ll R^{\delta} .
$$

Hence 


$$
\begin{aligned}
& \sum_{r \sim R} \sum_{q \leq P} \max _{(a, q)=1} \max _{(b, r)=1} \max _{|\lambda| \leq 1 /(q Q)}|K| \\
& \ll R^{\delta} \sum_{k_{1} \leq 2 R} \sum_{\substack{k_{2} \leq P \\
\left(k_{1}, k_{2}\right)=1}} \frac{1}{k_{1} k_{2}^{1 / 2}} \\
& \quad \times \max _{|\lambda| \leq 1 /\left(k_{2} Q\right)} \sum_{\substack{\chi_{1} \bmod k_{1} \\
\chi_{1} \neq \chi_{1}^{0}}}^{*} \sum_{\chi_{2} \bmod k_{2}}^{*}\left|\sum_{n \leq N} \chi_{1} \chi_{2}(n) \Lambda(n) e(n \lambda)\right| .
\end{aligned}
$$

One thus concludes from (5.6)-(5.9) that

$$
\begin{aligned}
& \sum_{r \sim R} \sum_{q \leq P} E^{*}(r, q) \\
= & \sum_{r \sim R} \sum_{q \leq P} \max _{(a, q)=1} \max _{(b, r)=1} \max _{|\lambda| \leq 1 /(q Q)}\left|E(r, q, a, b)-f(r, q, a, b) \sum_{n \leq N} e(n \lambda)\right| \\
\ll & \sum_{r \sim R} \sum_{q \leq P} \max _{(a, q)=1} \max _{(b, r)=1} \max _{|\lambda| \leq 1 /(q Q)}\left|I-f(r, q, a, b) \sum_{n \leq N} e(n \lambda)\right| \\
& +\sum_{r \sim R} \sum_{q \leq P} \max _{(a, q)=1} \max _{(b, r)=1|\lambda| \leq 1 /(q Q)} \max _{|J|}|J| \\
& +\sum_{r \sim R} \sum_{q \leq P} \max _{(a, q)=1} \max _{(b, r)=1|\lambda| \leq 1 /(q Q)}|K|+R P L^{2}+P^{3 / 2} L^{3} \\
\ll & \frac{L^{9}}{R^{1 / 2}} \sum_{k \sim U} \max _{|\lambda| \leq 1 /(U Q)} \sum_{\chi \bmod k}^{*}\left|\sum_{n \leq N} \chi(n) \Lambda(n ; \chi) e(n \lambda)\right| \\
& +\frac{R^{\delta}}{R U^{1 / 2}} \sum_{k \sim U R} \max _{|\lambda| \leq 1 /(U Q)} \sum_{\chi \bmod k}^{*}\left|\sum_{n \leq N} \chi(n) \Lambda(n ; \chi) e(n \lambda)\right| \\
& +R P L^{2}+P^{3 / 2} L^{3},
\end{aligned}
$$

where

$$
U \leq P=R^{2} L^{C}
$$

By (4.3) with $z=1$, the first term on the right-hand side of (5.10) is admissible if

$$
U \leq N^{1 / 3} L^{-D}, \quad \frac{1}{U Q} \leq U^{-3} L^{-D}
$$

Taking $z=R^{1 / 2-\delta}$ in (4.3), we see that the second term on the right-hand 
side of (5.10) is admissible if

$$
R U \leq R^{1 / 3-\delta} N^{1 / 3} L^{-D}, \quad \frac{1}{U Q} \leq R^{1-\delta}(R U)^{-3} L^{-D} .
$$

In view of the definitions of $Q$ and $U$ (see (2.2) and (5.11)), the optimal choice of $R$ satisfying (5.12) and (5.13) is

$$
R \leq N^{1 / 8-\varepsilon}
$$

as stated in (2.1). This proves the lemma.

6. The major arcs. In this section we give

Pro of of (2.5). In the course of the proof, the following elementary estimate will be used: If $A_{j}=B+C, j=1,2,3$, then

$$
A_{1} A_{2} A_{3}=B^{3}+C\left(A_{1}^{2}+B^{2}+A_{1} B\right)=B^{3}+O\left(|C| \cdot\left|A_{1}\right|^{2}+|C| \cdot|B|^{2}\right) .
$$

If $\alpha \in E_{1}(R)$, then for $j=1,2,3$,

$$
S\left(\alpha ; r, b_{j}\right)=f\left(r, q, a, b_{j}\right) \sum_{n \leq N} e(n \lambda)+O\left(E^{*}(r, q)\right) .
$$

Applying (6.1), one has

$$
\begin{aligned}
I_{1}(r):= & \int_{E_{1}(R)} S\left(\alpha ; r, b_{1}\right) S\left(\alpha ; r, b_{2}\right) S\left(\alpha ; r, b_{3}\right) e(-N \alpha) d \alpha \\
= & \sum_{q \leq P} \sum_{\substack{a=1 \\
(a, q)=1}}^{q}\left\{f\left(r, q, a, b_{1}\right) f\left(r, q, a, b_{2}\right) f\left(r, q, a, b_{3}\right) e\left(-\frac{a N}{q}\right)\right. \\
& \times \int_{|\lambda| \leq 1 /(q Q)}\left(\sum_{n \leq N} e(n \lambda)\right)^{3} e(-N \lambda) d \lambda \\
& +O\left(E^{*}(r, q) \int_{|\lambda| \leq 1 /(q Q)}\left|S\left(\frac{a}{q}+\lambda ; r, b_{1}\right)\right|^{2} d \lambda\right) \\
& \left.+O\left(\frac{E^{*}(r, q)}{\varphi^{2}(r q / h)} \int_{|\lambda| \leq 1 /(q Q)}\left|\sum_{n \leq N} e(n \lambda)\right|^{2} d \lambda\right)\right\} .
\end{aligned}
$$

The third integral on the right-hand side above is trivially $\ll N$. While the second integral, when summed over $a$, can be estimated as

$$
\sum_{\substack{a=1 \\(a, q)=1}}^{q} \int_{|\lambda| \leq 1 /(q Q)}\left|S\left(\frac{a}{q}+\lambda ; r, b_{1}\right)\right|^{2} d \lambda \leq \int_{0}^{1}\left|S\left(\frac{a}{q}+\lambda ; r, b_{1}\right)\right|^{2} d \lambda \leq \frac{N}{r} .
$$


On using the estimate

$$
\sum_{n \leq N} e(n \lambda) \ll \min (N, 1 /\|\lambda\|),
$$

one sees that the first integral is

$$
\begin{aligned}
\int_{-1 / 2}^{1 / 2}\left(\sum_{n \leq N} e(n \lambda)\right)^{3} e(-N \lambda) d \lambda+O\left(\int_{1 /(q Q)}^{1 / 2} \lambda^{-3} d \lambda\right) \\
=\sum_{\substack{n_{1}+n_{2}+n_{3}=N \\
1 \leq n_{j} \leq N}} 1+O\left((q Q)^{2}\right)=\frac{1}{2} N^{2}+O\left(N^{2} L^{-C}\right) .
\end{aligned}
$$

We thus have

$$
\begin{aligned}
I_{1}(r)= & \left(\frac{1}{2} N^{2}+O\left(N^{2} L^{-C}\right)\right) \\
& \times \sum_{q \leq P} \sum_{\substack{a=1 \\
(a, q)=1}}^{q} f\left(r, q, a, b_{1}\right) f\left(r, q, a, b_{2}\right) f\left(r, q, a, b_{3}\right) e\left(-\frac{a N}{q}\right) \\
& +O\left(\frac{N}{r} \sum_{q \leq P} E^{*}(r, q)\right) .
\end{aligned}
$$

We now consider the singular series

$$
\sum_{q=1}^{\infty} \sum_{\substack{a=1 \\(a, q)=1}}^{q} f\left(r, q, a, b_{1}\right) f\left(r, q, a, b_{2}\right) f\left(r, q, a, b_{3}\right) e\left(-\frac{a N}{q}\right) .
$$

For $(q / h, h)>1$, one has $f\left(r, q, a, b_{j}\right)=0, j=1,2,3$, by $(6.2)$, hence the series converges absolutely to 0 . For $(q / h, h)=1$, the series reduces to

$$
\frac{1}{\varphi^{3}(r)} \sum_{\substack{q=1 \\(q / h, h)=1}}^{\infty} \frac{\mu(q / h)}{\varphi^{3}(q / h)} \sum_{\substack{a=1 \\(a, q)=1}}^{q}\left(\frac{a\left(b_{1}+b_{2}+b_{3}\right) t}{h}-\frac{a N}{q}\right) .
$$

It was proved by Rademacher $[\mathrm{R}]$ that if $N$ is odd and $\mathbf{b} \in \mathcal{B}(N, r)$, then the above series converges absolutely and equals $\sigma(N ; r)$ defined as in (1.7). One therefore has

$$
I_{1}(r)-\sigma(N ; r) \frac{N^{2}}{2} \ll \frac{N^{2}}{\varphi^{2}(r) L^{C}}+\frac{N}{r} \sum_{q \leq P} E^{*}(r, q),
$$

and consequently,

$$
\sum_{r \sim R} r \max _{\left(b_{j}, r\right)=1}\left|I_{1}(r)-\sigma(N ; r) \frac{N^{2}}{2}\right| \ll N^{2} L^{-A},
$$

if $C$ is sufficiently large. This proves (2.5), hence Theorem 2 . 
Acknowledgements. The authors would like to thank Professor Pan Chengdong for constant encouragement. Also, we are indebted to the referee for valuable suggestions.

\section{References}

[A] R. Ayoub, On Rademacher's extension of the Goldbach-Vinogradov theorem, Trans. Amer. Math. Soc. 74 (1953), 482-491.

[B] A. Balog, The prime $k$-tuplets conjecture on average, in: Analytic Number Theory (Allerton Park, Ill.), Birkhäuser, 1990, 47-75.

[BP] A. Balog and A. Perelli, Exponential sums over primes in an arithmetic progression, Proc. Amer. Math. Soc. 93 (1985), 578-582.

[D] H. Daven port, Multiplicative Number Theory, revised by H. L. Montgomery, Springer, 1980.

[HL] G. H. Hardy and J. E. Littlewood, Some problems of "partitio numerorum" III: On the expression of a number as a sum of primes, Acta Math. 44 (1923), $1-70$.

[La] A. F. Lavrik, The number of $k$-twin primes lying on an interval of a given length, Dokl. Akad. Nauk SSSR 136 (1961), 281-283 (in Russian); English transl.: Soviet Math. Dokl. 2 (1961), 52-55.

[Li] J. Y. Liu, The Goldbach-Vinogradov theorem with primes in a thin subset, Chinese Ann. Math., to appear.

[LT] M. C. Liu and K. M. Tsang, Small prime solutions of linear equations, in: Théorie des Nombres, J.-M. De Koninck and C. Levesque (eds.), de Gruyter, Berlin, 1989, 595-624.

[MP] H. Maier and C. Pomerance, Unusually large gaps between consecutive primes, Trans. Amer. Math. Soc. 332 (1990), 201-237.

[Mi] H. Mikawa, On prime twins in arithmetic progressions, Tsukuba J. Math. 16 (1992), 377-387.

[Mo] H. L. Montgomery, Topics in Multiplicative Number Theory, Lecture Notes in Math. 227, Springer, 1971.

[R] H. Rademacher, Über eine Erweiterung des Goldbachschen Problems, Math. Z. 25 (1926), 627-660.

[T] E. C. Titchmarsh, The Theory of the Riemann Zeta-Function, 2nd ed., revised by D. R. Heath-Brown, Oxford Univ. Press, 1986.

[Va] R. C. Vaughan, An elementary method in prime number theory, in: Recent Progress in Analytic Number Theory, H. Halberstam and C. Hooley (eds.), Vol. 1, Academic Press, 1981, 341-348.

[Vi] I. M. Vinogradov, Some theorems concerning the theory of primes, Mat. Sb. (N.S.) 2 (1937), 179-195.

[W] D. Wolke, Some applications to zero-density theorems for L-functions, Acta Math. Hungar. 61 (1993), 241-258.

[Zh] T. Zhan, On the representation of odd number as sums of three almost equal primes, Acta Math. Sinica (N.S.) 7 (1991), 259-275.

[ZL] T. Zhan and J. Y. Liu, A Bombieri-type mean-value theorem concerning exponential sums over primes, Chinese Sci. Bull. 41 (1996), 363-366. 
[Zu] A. Zulauf, Beweis einer Erweiterung des Satzes von Goldbach-Vinogradov, J. Reine Angew. Math. 190 (1952), 169-198.

Department of Mathematics

Shandong University

Jinan, Shandong 250100

P.R. China

E-mail: zhantao@sdu.edu.cn

Received on 8.6.1996

and in revised form on 6.12.1996 